\title{
ALICE ITS Operational Experience
}

\section{Deplano* on behalf of the ALICE Collaboration}

Nikhef, The Netherlands

E-mail: Caterina.Deplano@cern.ch

ALICE, A Large Ion Collider Experiment, is conceived to study the physics of strongly interacting matter and the properties of the Quark-Gluon Plasma produced in ultra-relativistic heavy-ion collisions at the CERN LHC.

The innermost detector of ALICE is the Inner Tracking System (ITS) which plays the essential role of primary and secondary vertex reconstruction. It is used for particle tracking and identification and contributes to the first level trigger. The ITS covers the pseudo-rapidity range $|\eta|<0.9$ and consists of six cylindrical layers of silicon detectors placed coaxially around the beam pipe. Three different technologies were selected to equip the ITS: pixel detectors for the two inner layers, drift detectors for the two central layers and strip detectors for the outer layers.

In this report the three detectors constituting the ITS are briefly described, the operational experience during RUN2 is summarised with a focus on the performance of the detector compared to RUN1 and on the interventions done during the long technical stop at the end of 2015.

The 25th International workshop on vertex detectors

September 26-30, 2016

La Biodola, Isola d'Elba, ITALY

* Speaker. 


\section{Introduction}

ALICE [1], A Large Ion Collider Experiment, is devoted to study the physics of strongly interacting matter and the properties of the Quark-Gluon Plasma produced in ultra-relativistic heavy-ion collisions at the LHC. The ALICE experimental program also includes $\mathrm{pp}$ and $\mathrm{p}-\mathrm{Pb}$ collisions, that are used to complement the observation of the dedicated pp experiments and to collect reference data.

The ALICE apparatus is composed of a central barrel and a forward muon arm, having a pseudorapidity range of $|\eta|<0.9$ and $2.5<|\eta|<4.0$ respectively. ALICE has an excellent particle identification capability up to $20 \mathrm{GeV} / c$, based on $\mathrm{d} E / \mathrm{d} x$, time of flight, transition and Cherenkov radiation, calorimetry and topological reconstruction.

The Inner Tracking System (ITS) [2] is the innermost detector of the central barrel and together with the Time Projection Chamber (TPC) it provides a robust tracking in a wide transverse momentum range $(0.1 \div 100 \mathrm{GeV})$. The low momentum cut-off is obtained minimizing the multiple scattering thanks to the low material budget, which is about $10 \% X_{0}$ for the ITS and the TPC. The ITS plays an essential role in primary and secondary vertex reconstruction. It is used for particle tracking and identification and contributes to the first level trigger.

A detailed description of the ITS is given in Section 2. In Sections 3 the ITS performance during RUN2 is presented. Finally, the ITS physics performance of tracking, particle identification and vertexing is described in Section 4.

\section{The Inner Tracking System}

The Inner Tracking System, shown in Figure 1, is composed of six cylindrical layers instrumented with three different detector technologies:

- SPD: Silicon Pixel Detector for the two inner layers

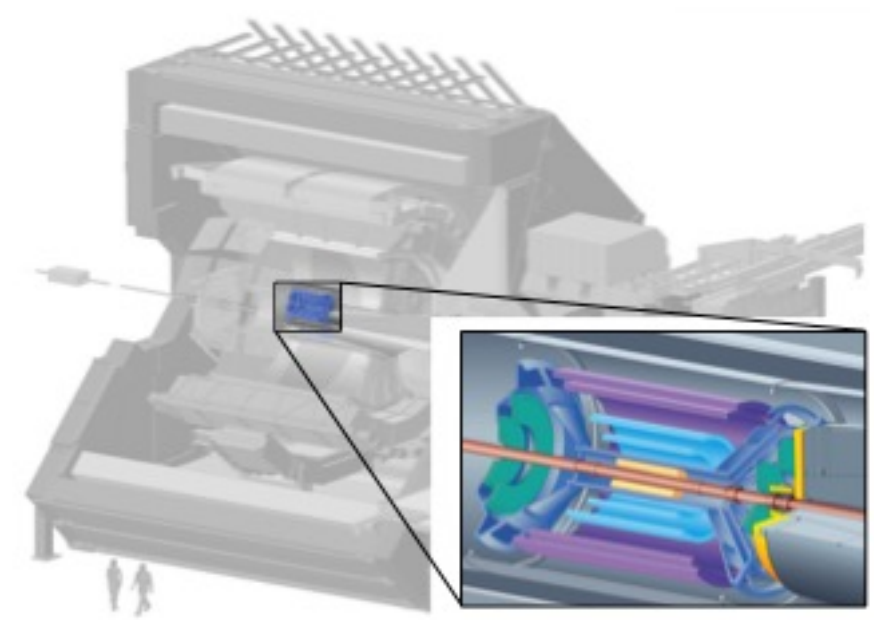

Figure 1: Zoom of the ITS layout in the center of the ALICE central barrel: SPD in yellow, SDD in blue, SSD in purple. 


\begin{tabular}{|l|c|c|c|}
\hline \hline Layer/Detector & 1-2/SPD & 3-4/SDD & $5-6 / \mathrm{SSD}$ \\
\hline Surface $\left(\mathrm{m}^{2}\right)$ & 0.21 & 1.31 & 5.0 \\
\# of Channels & $9.8 \mathrm{M}$ & $133 \mathrm{k}$ & $2.6 \mathrm{M}$ \\
Cell size $\left(\mu \mathrm{m}^{2}\right)$ & $50 \times 425$ & $202 \times 294$ & $95 \times 40 \mathrm{k}$ \\
\hline Spatial Precision $(\mu \mathrm{m})$ & & & \\
$r \phi$ & 12 & 35 & 20 \\
$z$ & 100 & 25 & 830 \\
\hline Power Dissipation $(\mathrm{W})$ & & & \\
Barrel + End-Cap & $1.38 \mathrm{k}$ & $1.8 \mathrm{k}$ & $2 \mathrm{k}$ \\
\hline
\end{tabular}

Table 1: Main ITS parameters per detector.

- SDD: Silicon Drift Detector for the two central layers

- SSD: Silicon double-sided $\mu$-Strip Detector for the two outer layers

The segmentation and the position of the six layers were chosen to limit the occupancy to few $\%$ in the high-multiplicity events of central $\mathrm{Pb}-\mathrm{Pb}$ collisions and to assure an efficient tracking. Inwards, the radial coverage is constrained by the beam vacuum tube; outwards, by the inner TPC vessel position and by the TPC track matching requirements. The material budget per layer is about $1 \% X_{0}$ for straight track perpendicular to the surface. The main ITS parameters are summarized in Tables 1 and 2. The four outer layers, SDD and SSD, have an analog readout used to extract the energy loss $\mathrm{d} E / \mathrm{d} x$ information which allows to use the ITS for standalone particle identification.

\subsection{Silicon Pixel Detector - SPD}

The SPD is made of hybrid pixel detectors with a binary output. The SPD is composed by 120 half-stave modules grouped in two half-barrels. Each half-barrel is divided in 10 semi-sectors containing 6 half-staves, which are composed by 2 ladders. Each ladder contains 1 sensor ( $200 \mu \mathrm{m}$ thick) and 5 readout chips ( $150 \mu \mathrm{m}$ thick) connected via bump-bonding. The sensor is a matrix of $256 \times 160 \mathrm{p}^{+} \mathrm{n}$ reversely biased diodes with pixel size $425 \times 50 \mu \mathrm{m}^{2}(z r \phi)$. The nominal bias voltage is $-50 \mathrm{~V}$. The SPD makes use of an evaporative $C_{4} F_{10}$ cooling system. The binary output is readout at $10 \mathrm{MHz}$.

\begin{tabular}{|l|c|c|c|c|c|c|}
\hline \hline Layer/Detector & 1/SPD & 2/SPD & 3/SDD & 4/SDD & 5/SSD & 6/SSD \\
\hline Radius $(\mathrm{cm})$ & 3.9 & 7.6 & 15.0 & 23.9 & 38.0 & 43.0 \\
Length $(\mathrm{cm})$ & 28.2 & 28.2 & 44.4 & 59.4 & 86.2 & 97.8 \\
Max Occupancy Central PbPb (\%) & 2.1 & 0.6 & 2.5 & 1.0 & 4.0 & 3.3 \\
Material Budget $\left(\% \mathrm{X} / \mathrm{X}_{0}\right)$ & 1.14 & 1.14 & 1.13 & 1.26 & 0.83 & 0.86 \\
\# of Ladders & 80 & 160 & 14 & 22 & 34 & 38 \\
\hline
\end{tabular}

Table 2: Main ITS parameters per layer. 


\subsubsection{SPD as trigger detector}

The SPD is the only ITS sub-detector which contributes to the ALICE first level trigger. Each pixel readout chip output the data corresponding to the fired pixels and one Fast-OR signal with the digital value 1 if at least one of the related 8192 pixels is fired. Data and Fast-OR are both recorded using a 4-events deep Multi-Event-Buffer and the readout strobed on the two separate lines must be matched in time with a time delay unit of $100 \mathrm{~ns}$. The main SPD novelty in Run 2 consisted in the exploitation of the SPD triggering capabilities with the High Multiplicity trigger (2015) as well as the Double Gap Diffractive trigger (2016), a topological trigger for dedicated pp event topologies [3]. The data collected by both triggers are under study.

\subsection{Silicon Drift Detector - SDD}

The SDD consists of 36 ladders, 14 placed in the SDD inner layer and 22 placed in the SDD outer layer. The ladders in the inner layer are equipped with 6 modules each, while 8 modules are used to instrument each ladder in the outer layer, for a total of 260 modules. Each module is composed of a silicon drift sensor ( $300 \mu \mathrm{m}$ thick) and two hybrid boards with the readout chips. An electrical drift field of about $500 \mathrm{~V} / \mathrm{cm}$, parallel to the surface of the sensor, is created by a central cathode strip biased $-1.8 \mathrm{kV}$. The collection anodes are 512 per sensor, spaced by $294 \mu \mathrm{m}$ and located on the two sides of the sensor, parallel to the beam axis. Three rows of 22 MOS charge injectors are used to monitor the drift velocity, which is stable around the value of $6.7 \mu \mathrm{m} / \mathrm{ns}$, at the beginning of each LHC fill. The analog information is digitalized by 10-bits ADCs at $20 \mathrm{MHz}$, already at the level of the front-end electronics, developed on CMOS $0.25 \mu \mathrm{m}$. The SDD makes use of a water cooling system.

\subsection{Silicon Strip Detector - SSD}

The SSD consist of 1698 modules, each one composed of 768 double-sided silicon strip sensors (300 $\mu \mathrm{m}$ thick) connected to 12 front-end chips. The analog signals are extracted from the detector and digitized by 12-bit ADCs directly in the readout-electronics placed just outside the ALICE solenoid magnet, in the cavern. The strips on the P-side and on the $\mathrm{N}$-side of the sensor are oriented respectively at an angle of $7.5 \mathrm{mrad}$ and $27.5 \mathrm{mrad}$ with respect to the beam axis. The strip orientations, approximately parallel to the ALICE solenoid magnetic field, ensure the best position resolution in the bending direction. The $\mathrm{p}^{+} \mathrm{n}$ reverse bias is optimized in the range $20 \div 80 \mathrm{~V}$. The SSD makes use of a water cooling system, shared with the SDD, and of an air dryer system.

\section{The ITS RUN2 Performance}

In Table 3 the RUN2 performance in terms of availability in data taking and acceptance is reported for each of the three ITS detectors. The values are compared with the RUN1 ones. The ITS detector's data taking time, relative to the total ALICE data taking time, improved with respect to RUN1 and this is mainly related to the consolidation activities:

- SPD Cooling: At the end of RUN1 several SPD modules were found to have an insufficient flow of the liquid freon, due to a partial clogging of the filters installed in the feeding lines 


\begin{tabular}{|l|c|c|}
\hline & Availability in data taking & Acceptance (\% of Modules) \\
\hline \hline SPD RUN1 & $96 \%$ & $92 \%$ \\
\hline SPD RUN2 & $99.6 \%$ & $93 \%$ \\
\hline \hline SDD RUN1 & $92 \%$ & $87 \%$ \\
\hline SDD RUN2 & $98.4 \%$ & $82.8 \%$ \\
\hline \hline SSD RUN1 & $96 \%$ & $91 \%$ \\
\hline SSD RUN2 & $98.6 \%$ & $91 \%$ \\
\hline \hline
\end{tabular}

Table 3: ITS availability in data taking and acceptance during RUN1 and RUN2.

close to the detector. The problem has been solved during LS1 (Long Shutdown) by drilling the filters in order to re-establish the correct flow [4].

- SDD Recovery Time: The SDD team improved the recovery time needed if one or more front-end chips lose the configuration (like baseline, thresholds, masks) during the run, caused by Single Event Upset (SEU) events. In order to outcome this problem the SDD readout boards were upgraded during LS1 with a more performing FPGA (Xilinx Virtex5). The new FPGA capability allowed to design a new firmware with an automatic procedure which event by event detects noisy chips end eventually reloads the configuration parameters. Since the beginning of 2015 the recovery time is about $800 \mu$ s and stopping and starting the run is no longer needed. Since September 2016 the information on the reconfigured chip is encoded in the data to save the statistical information of the SEU events recovered.

- SSD Recovery Time: The SSD team improved the procedure to recover the errors related to SEU events affecting the readout electronics, which is placed in the cavern. Since November 2015 the recovery time improved from 20 minutes to less then 5 minutes. The SSD SEU events is the main ITS issue during RUN2, and it is described in Section 3.1.

For what concerns the acceptance, expressed in terms of number of active modules, all the ITS detectors show a good stability since the beginning of RUN2. At the same time the SDD had to exclude some modules during the RUN2 recommissioning because of front-end electronics problems (HV, noise, shorts, disconnected channels) which are not recoverable due to the impossibility to access the detector.

\subsection{SSD Single Event Upset events}

The SSD Front End Read Out Module (FEROM) [5] electronics is located in the cavern, just outside the solenoid magnet. The analog data coming from the end-cap electronics are digitized, time tagged, zero-suppressed and sent via optical link from the FEROM to the local data concentrator. The components affected from SEU events are mainly the SRAM FPGAs. Table 4 lists the FPGA and the PROM devices under use. The signature of the SSD SEU events can be described as follows.

- The online data quality plots show a data size over-threshold which is not correlated to the occupancy and which is not recovered with a new pedestal calculation. 
- The error appears in presence of the beam.

- The error can affect the data transmitted via the optical links.

- Recovery is possible only by reloading the FPGA firmware.

According to simulation [5] the FEROM system is exposed to a Total Ionizing Dose (TID) of about $4 \cdot 10^{-4}$ Gray in 10 years of data taking. Many mitigation techniques were implemented in the FEROM electronics design, like the Data Parity Check, the JTAG used for FPGA reconfiguration, the polyswitch fuses to protect power supply from latch-up. During LS1 the PROMs in the FEROM were replaced with radiation tolerant PROMs and the FPGAs firmware was upgraded for faster FPGA reload. Moreover since September 2015 the software procedure to recover from a SEU events has been automatized to minimize the data taking stopping time, being currently about 5 minutes.

The RUN2 SEU events in SSD are correlated to the ALICE integrated luminosity and to the fluence measured at the FEROM crate location. At the same time the statistics collected (18 SEU events from the beginning of RUN2 until September 2016) is too low to make accurate predictions.

\section{ITS Physics Performance}

The ITS plays a crucial role in the reconstruction of the interaction (primary) and secondary vertices. The algorithm used to perform this task is based on an iterative procedure using tracks reconstructed in the whole ALICE central barrel with the ITS and the TPC. To extend the transverse momentum acceptance down to $0.1 \mathrm{GeV} / c$ and to recover tracks not reconstructed in the TPC, an ITS-standalone tracking algorithm was developed. The efficiency of the prolongation of the ITS standalone tracks to the TPC, for tracks with at least 2 points in the ITS, is above $95 \%$ and is almost constant in the transverse momentum range.

The resolution of the track transverse impact parameter $d_{0}$, calculated as the distance of closest approach of a given track to the vertex, allows to define the tracking precision of the system. In Figure 2 (left) the impact parameter resolution in the $x-y$ plane, for identified ITS-TPC tracks, is reported as function of the transverse momentum for three different collision systems [6]. For all of them the $d_{0}$ resolution is about $60 \mu \mathrm{m}$ at $1 \mathrm{GeV} / c$; the contribution of the primary vertex resolution is not subtracted. The precise measurement of the track impact parameter and the capability to separate the primary and secondary vertices, allowed the inclusive reconstruction of various rare particles via their hadronic decays.

The ITS contributes to the ALICE particle identification measuring the ionizing energy loss $\mathrm{d} E / \mathrm{d} x$ in the four outermost layers via the readout of the analog signal proportional to the charge

\begin{tabular}{|c|c|c|}
\hline Device Type & Device Model & \# of exposed devices \\
\hline SRAM FPGA & Xilixn XC2V1000FG456 (VIRTEXII) & 8 \\
SRAM FPGA & Xilixn XC2S50EPQ208 (SPARTANII) & 216 \\
RAD HARD PROM & Xilinx XQR18V04CC44M & 16 \\
\hline
\end{tabular}

Table 4: FPGA and PROM devices exposed to radiation on the SSD FEROM system. 

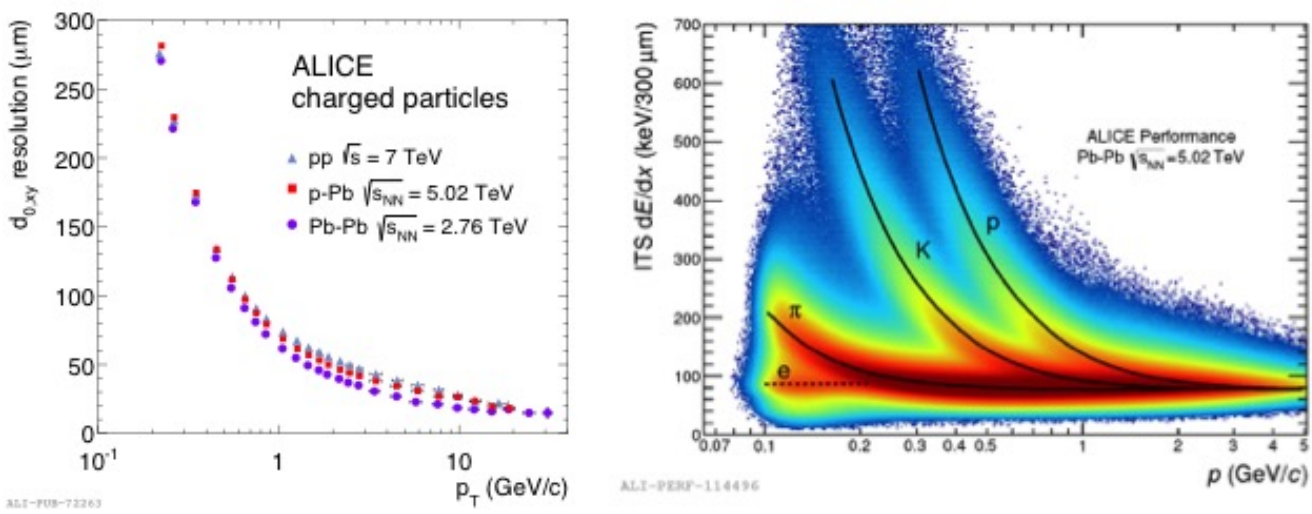

Figure 2: Left: Impact parameter resolution in the $x-y$ plane for identified ITS-TPC tracks plotted as a function of the transverse momentum for three different collision systems. Right: Ionizing energy loss $\mathrm{d} E / \mathrm{d} x$ for charged particles as a function of their momentum in $\mathrm{Pb}-\mathrm{Pb}$ collisions at $5.02 \mathrm{TeV}$; ITS standalone tracking.

released in the detector layer [7]. Figure 2 (right) shows an example of the mean energy deposition as a function of the momentum measured in $\mathrm{Pb}-\mathrm{Pb}$ collisions at $5.02 \mathrm{TeV}$. The ITS particle identification combined with the standalone tracking allows for a K- $\pi$ and a K-p separation in the range of $(0.1 \div 0.45) \mathrm{GeV} / c$ and $(0.1 \div 1) \mathrm{GeV} / c$ respectively [7]

\section{Summary and Conclusions}

The ALICE Inner Tracking System has been successfully in operation during RUN1 and since the beginning of RUN2 with high efficiency and a stable participation in $\mathrm{pp}, \mathrm{Pb}-\mathrm{Pb}$ and $\mathrm{p}-\mathrm{Pb}$ data taking. During RUN2 new procedures have been implemented to minimize the recovery time related to Single Event Upset events, and the SPD contributes to the first level trigger with new algorithms. SPD, SDD and SSD perform remarkably well and according to the specifications. The physics performance is in agreement with the design requirements and is stable with time.

In order to extend the heavy-quark studies to the decay of charmed baryons and beauty hadrons in RUN3, an upgrade of the system is under development [8].

\section{References}

[1] ALICE Collaboration, The ALICE experiment at the CERN LHC, JINST 3, S08002 (2008).

[2] ALICE Collaboration, Technical Design Report of the Inner Tracking System (ITS), CERN-LHCC-99-012 (1999).

[3] A. Mastroserio, Operational Experience with the ALICE Pixel detector, in proceedings of PIXEL2016 conference, prepared for submission to JINST, (2016).

[4] D. Colella, ALICE ITS: the RUN1 to RUN2 transition and recent operational experience, in proceedings of VERTEX2015 conference, PoS VERTEX2015 (2015) 003.

[5] M. J, Rossewij et al., FEROM, the ALICE SSD read out system, proceeding in 9th Workshop on Electronics for LHC Experiments, (2003) [10.5170/CERN-2003-006.157]. 
[6] ALICE Collaboration, Performance of the ALICE experiment at the CERN LHC, Int. Mod. Phys., A29 (2014) 1430044.

[7] ALICE Collaboration, Centrality dependence of $\pi$, K, and p production in $\mathrm{Pb}$-Pb collisions at $\sqrt{s_{N N}}$ $=2.76$ TeV, Phys. Rev. C 88 (2013) 044910.

[8] ALICE Collaboration, Technical Design Report for the Upgrade of the ALICE Inner Tracking System, CERN-LHCC-2013-024 (2013). 